\title{
A Comparative Study of Impedance Measurement Techniques for Structural Health Monitoring Applications
}

\author{
Danilo Ecidir Budoya ${ }^{\circledR}$, Member, IEEE, and Fabricio Guimarães Baptista ${ }^{\circledR}$, Member, IEEE
}

\begin{abstract}
Structural health monitoring (SHM) systems are scientifically and economically relevant as methods of detecting structural damage to various types of structures, thus increasing safety and reducing maintenance costs. Among the various principles of damage detection, the electromechanical impedance (EMI) method is based on the electrical impedance measurement of piezoelectric transducers attached to the monitored structure. Here, the accuracy and precision of the measurement system are fundamental for the correct diagnosis of the structure. Therefore, this paper performs a comparative analysis of two impedance measurement techniques for damage detection that are typically used in commercial impedance analyzers and other alternative measurement systems: 1) transient-state measurements using a sweep excitation signal and 2) steady-state measurements using a pure sinusoidal signal for each excitation frequency. Tests were performed with resistive and capacitive loads with known values and a piezoelectric transducer fixed to an aluminum bar representing a monitored structure. The two techniques were compared based on the accuracy, precision, and time required for the measurements. The results highlight the important features of each technique that should be considered for the development of impedance-based SHM systems and the correct diagnosis of monitored structures.
\end{abstract}

Index Terms-Electromechanical, impedance, measurement, piezoelectric transducers, structural health monitoring (SHM).

\section{INTRODUCTION}

$\mathbf{T}$ HE detection of structural damage at an early stage is of global interest; consequently, such detection has become a prominent field of research in several academic and industrial segments. Civil infrastructure and large means of transport, such as bridges, aircraft, ships, trains, and dams, are some examples of structures that can be monitored. Damage to these structures, including cracks, corrosion, and loosening of bolted connections, can change their normal operation and, consequently, endanger their users. For this purpose, structural health monitoring (SHM) systems have been researched and developed in recent years [1], [2]. These systems monitor

Manuscript received May 22, 2017; revised December 20, 2017; accepted December 25, 2017. Date of publication February 5, 2018; date of current version March 8, 2018. This work was supported by the São Paulo Research Foundation (FAPESP) under Grant 2015/02500-6 and Grant 2015/23272-1. The Associate Editor coordinating the review process was Dr. Yuhua Cheng. (Corresponding author: Fabricio Guimarães Baptista.)

The authors are with the São Paulo State University (UNESP), School of Engineering, Bauru, Department of Electrical Engineering, Laboratory of Transducers and Data Acquisition, Bauru 17033-360, Brazil (e-mail: danilo.budoya@feb.unesp.br; fabriciogb@feb.unesp.br).

Color versions of one or more of the figures in this paper are available online at http://ieeexplore.ieee.org.

Digital Object Identifier 10.1109/TIM.2018.2792854 structures in real time or periodically to detect and locate damage. In addition to enhancing user safety, SHM systems also help reduce maintenance costs because damage can be detected at an early stage.

Most structures cannot accommodate frequent operational interruptions for monitoring of detection of uncertain damage. Therefore, SHM systems must use an embedded damage detection method that is minimally invasive to the structure and allows monitoring under normal operating conditions. Methods that meet these requirements are known as nondestructive evaluation or nondestructive testing (NDT) methods; NDT techniques reported in the literature include acoustic emission [3]-[5], Lamb waves [6], [7], eddy currents [8] and eddy current pulsed thermography [9], Doppler radar [10], and guided ultrasonic waves [11]. In addition, new methods and sensors [12]-[14] continue to be developed to ensure precision, accuracy, and low-cost production of reliable SHM systems.

Among the various methods available for damage detection, the electromechanical impedance (EMI) method [15]-[17] uses piezoelectric transducers simultaneously operating as an actuator and sensor. The basic methodology of the EMI method is to attach the piezoelectric transducer to the structure to be monitored. Due to the piezoelectric effect, the mechanical impedance of the monitored structure becomes coupled to the electrical impedance of the transducer. Structural damage changes the mechanical impedance of the structure, which causes a corresponding variation in the electrical impedance of the transducer. Therefore, the structure can be monitored by measuring and analyzing the electrical impedance of the transducer.

Damage is typically characterized using damage indices. The most common damage indices in the literature are based on the comparison between two electrical impedance signatures, where one of them is obtained when the structure is in a state that is considered healthy. Since the damage detection is based on the comparison between two impedance signatures, the accuracy and precision of the measurement system are fundamental to ensure the correct diagnosis of the monitored structure.

In the past, the electrical impedance measurements were performed primarily with commercial impedance analyzers such as HP 4194A and HP 4294A. These instruments continue to be used in recent studies [18], [19] of the EMI method because of their favorable accuracy and precision. However, these instruments are expensive and have many features not 
required by the EMI method, in addition to being bulky, which makes them ill-suited practical applications.

Therefore, to overcome the practical disadvantages of the conventional instruments, alternative measurement systems have been proposed. These systems are mainly based on microcontroller [20] or multifunctional data acquisition (DAQ) devices [21]. Recently, many impedance measurement systems have been developed using the AD5933 chip [22] from Analog Devices (Norwood, MA, USA), which is a high-precision impedance converter solution that includes a direct digital synthesizer core and a digital-to-analog converter (DAC). These devices generate a sinusoidal signal for the excitation of the unknown impedance being tested, and an analog-todigital converter (ADC) is used to sample the response signal. The discrete Fourier transform (DFT) of the response signal is processed by an onboard digital signal processor engine, which returns a real and imaginary data word of the unknown impedance at each output frequency.

Measurement systems based on the AD5933 chip can be optimized to include wireless communication and the measurements of multiple sensors. Several applications have used measurement systems based on this chip, such as the detection of structural damage in SHM [23], [24], bioelectrical impedance analysis [25], and detection of protein biomarkers [26].

The commercial impedance analyzers and these alternative systems proposed in the literature typically use one of the following measurement techniques: 1) transient-state measurements, which use a sweep signal such as a chirp [27] to excite the transducer (or other device under test) over a wide frequency band in a short time or 2) steady-state measurements, which use a pure sine wave signal for each frequency, requiring several steps and a longer duration to complete the measurement.

This paper aimed to perform a comparative analysis between these two measurement techniques because the impedance measurement is critical in impedance-based SHM systems since structural damage detection is performed by comparing two impedance signatures. Therefore, highly accurate and precise measurements are critical for the correct diagnosis of the monitored structure. In addition, other factors, such as the time required for the measurements, should be considered in the development of embedded SHM systems for real-time monitoring.

Therefore, this paper presents important features of the two techniques that may assist readers in choosing the most appropriate measurement system for SHM or other applications that require impedance measurement. The remainder of this paper is organized as follows. The principles of the EMI method and the measurement techniques are presented in Sections II and III, respectively. The experimental configuration used to compare the two techniques is presented in Section IV. The results are presented and discussed in Section V, and the conclusions are presented in Section VI.

\section{EMI PRINCIPLE}

The basic configuration of the EMI method is shown in Fig. 1, which shows a piezoelectric transducer attached to the structure to be monitored.

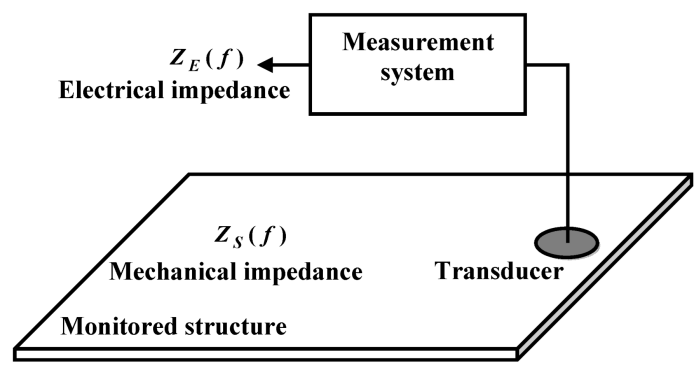

Fig. 1. Basic configuration of the EMI method.

As shown in Fig. 1, the measurement system excites the transducer at an appropriate frequency $(f)$ while simultaneously measuring its electrical impedance $\left(Z_{E}(f)\right)$. The most commonly used transducers in the EMI method are lead zirconate titanate (PZT) ceramics (also known as piezoelectric wafer active sensors [28]), macrofiber composites [29], and piezoelectric diaphragms [30], [31]. These transducers are lightweight, small, and thin, allowing multiple transducers to be attached to structures without significantly altering their mechanical properties.

The relationship between the electrical impedance $\left(Z_{E}(f)\right)$ of a piezoelectric transducer and the mechanical impedance $\left(Z_{S}(f)\right)$ of a structure has been extensively studied, and based on the basic constitutive equations of the piezoelectric material, several researchers have proposed various electromechanical models. A simplified version of one of the best-known 1-D models commonly used in the literature to show the interaction between the electrical and mechanical quantities in the EMI method using a PZT ceramic is [32]

$$
Z_{E}(f)=\frac{1}{j 2 \pi f C}\left(1-\frac{d_{31}^{2}}{s_{11}^{E} \varepsilon_{33}^{T}} \frac{Z_{S}(f)}{Z_{S}(f)+Z_{P}(f)}\right)^{-1}
$$

where $Z_{E}(f)$ is the electrical impedance of the transducer at the frequency $f ; Z_{P}(f)$ is the mechanical impedance of the transducer; $Z_{S}(f)$ is the mechanical impedance of the monitored structure; $C$ is the static capacitance of the transducer; $d_{31}, s_{11}^{E}$, and $\varepsilon_{33}^{T}$ are the piezoelectric, elastic compliance, and dielectric constants, respectively, of the piezoelectric material, where the superscripts $T$ and $E$ indicate constant stress and constant electric field, respectively, and the subscripts 1 and 3 represent the axes of the natural coordinate system of the piezoelectric material under the 1-D assumption; and $j$ is the unit imaginary number.

According to (1), any variation in the mechanical impedance $\left(Z_{S}(f)\right)$ of the monitored structure caused by damage results in a corresponding variation in the electrical impedance $\left(Z_{E}(f)\right)$ of the transducer. Therefore, structural damage can be detected and quantified by comparing two electrical impedance signatures using damage indices. The electrical impedance is complex, and the real part is typically used to calculate the damage indices since it is more sensitive to damage and less sensitive to temperature variations [1]. One of the most commonly reported damage indices in the literature to be considered in this paper is the root-mean-squared deviation (RMSD) 


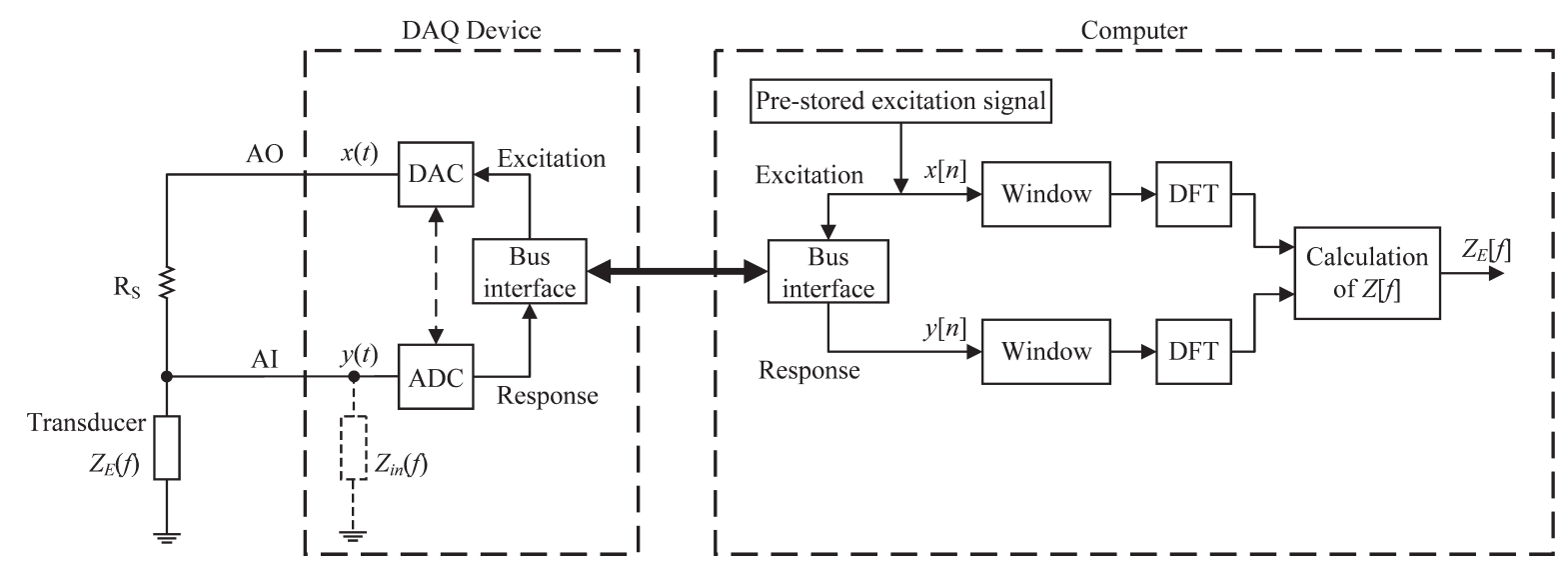

Fig. 2. Basic configuration used in the analysis of the measurement techniques, adapted from [21].

index, which is given by [1]

$$
\operatorname{RMSD}=\sum_{f=f_{I}}^{f_{F}} \sqrt{\frac{\left[\operatorname{Re}\left(Z_{2}(f)\right)-\operatorname{Re}\left(Z_{1}(f)\right)\right]^{2}}{\operatorname{Re}\left(Z_{1}(f)\right)^{2}}}
$$

where $\operatorname{Re}\left(Z_{1}(f)\right)$ is the real part of the impedance signature obtained for the structure considered healthy, also known as the baseline; $\operatorname{Re}\left(Z_{2}(f)\right)$ is the real part of the impedance signature after possible damage; and RMSD is the index calculated in the frequency range with initial frequency $f_{I}$ and final frequency $f_{F}$.

Ideally, disregarding environmental effects such as temperature, vibration, and noise, the impedance signatures and, consequently, the damage indices should vary only due to structural damage. Therefore, as already stated, the quality of the measurement system is fundamental to guarantee satisfactory reproducibility of the electrical impedance signatures and to avoid variations in the damage indices, which is essential for the correct diagnosis of the monitored structure. The two measurement techniques analyzed and compared in this paper are presented in Section III.

\section{iII. Measurement Techniques}

As mentioned in Section I, two techniques for exciting a piezoelectric transducer or other device are commonly used in commercial impedance analyzers and alternative measurement systems. In this section, the two measurement techniques are presented.

\section{A. Steady-State Measurement}

The steady-state measurement technique is similar to that used by commercial impedance analyzers and many alternative systems, especially systems based on the AD5933 chip [22]-[26]. In this technique, the electrical impedance of the transducer is measured step by step using a sinusoidal signal for each frequency of interest.

In this paper, the analysis of the measurement techniques was performed using a multifunctional DAQ device connected to a personal computer, similar to the configuration reported in [21]. The block diagram of the measurement system is shown in Fig. 2.
According to Fig. 2, the excitation signal $(x(t))$ is generated by an analog output (AO) of a DAC, which excites the transducer connected in series with a resistor $\left(R_{S}\right)$, resulting in a voltage divider. The response signal $(y(t))$ from the transducer is sampled by the analog input (AI) of an ADC synchronously with the excitation signal. The signals $x[n]$ and $y[n]$ are, respectively, the excitation and response signals in discrete time.

Commercial measuring instruments typically use autobalanced bridges or other techniques to maintain the amplitude of the excitation and response signals constant regardless of the impedance of the transducer or load being tested. Solutions similar to the voltage divider consisting of the resistor $\left(R_{S}\right)$ connected in series with the transducer have been used in several studies [33]-[35] because of its simplicity and versatility, although it has the disadvantage of not keeping the amplitude of the response signal constant regardless of the frequency $(f)$ at which the impedance is measured. Although from a strictly electrical point of view, the measured impedance does not depend on the signal amplitude, a lowexcitation signal can reduce the sensitivity to damage in SHM applications, particularly for large monitored structures. The variation of the signal amplitude can be minimized by choosing the appropriate resistor according to the analyzed frequency range or by adding a capacitor in series with the resistor to reduce the impedance mismatch, as reported in [33].

For steady-state measurements, the excitation signal is a pure sinusoidal wave $x(t)=A \sin \left(2 \pi f_{i} t\right)$ with amplitude $A$ and fixed frequency $f_{i}$. It is not necessary to sample this signal at each measurement because it is known and generated by software. However, it is recommended that the excitation signal be sampled and prestored to minimize the effects of the DAC, similar to the procedure followed in [21]. This approach increases the accuracy of the measurements and is implemented by connecting the input (AI) directly to the output (AO) of the DAQ device. Another advantage of this solution is that it allows a higher sampling rate on multiplexed DAQ devices because only one AI is required.

The impedance measured at each frequency is given by

$$
Z_{T}\left[f_{i}\right]=\frac{R_{S}}{\frac{X\left[f_{i}\right]}{Y\left[f_{i}\right]}-1}
$$


where $X\left[f_{i}\right]$ and $Y\left[f_{i}\right]$ are the DFT of the discrete time excitation signal $(x[n])$ and response signal $(y[n])$, respectively, at the frequency $f_{i}$, and $R_{S}$ is the resistor connected in series with the transducer, as shown in Fig. 2. Since the interest is in the specific frequency $f_{i}$, it is important to apply a window on the $x[n]$ and $y[n]$ signals, as shown in Fig. 2, before computing the DFT to minimize the effects of other frequencies resulting from the truncation of the signals.

The impedance $Z_{T}\left[f_{i}\right]$ calculated in (3) includes the input impedance $\left(Z_{\text {in }}\left[f_{i}\right]\right)$ of the DAQ device, which must be considered depending on the hardware and frequency range at which the impedance of the transducer is being measured. Therefore, considering that the connection wires are short and their resistances can be disregarded, the electrical impedance of the transducer $\left(Z_{E}\left[f_{i}\right]\right)$ can be accurately calculated as

$$
Z_{E}\left[f_{i}\right]=\frac{Z_{\text {in }}\left[f_{i}\right] Z_{T}\left[f_{i}\right]}{Z_{\text {in }}\left[f_{i}\right]-Z_{T}\left[f_{i}\right]}
$$

The input impedance $\left(Z_{\text {in }}\left[f_{i}\right]\right)$ can be calculated at each frequency $f_{i}$ according to the characteristics provided by the DAQ device manufacturer. A more practical and effective way, which was used in this paper, is to perform the measurement with the transducer (or load) disconnected. In this condition, the impedance provided by the measuring system will be the input impedance.

Because the measurements are performed step by step for each frequency, a large number of iterations may be required to obtain impedance signatures for large frequency bands and small frequency steps. The simplified flowchart of this measurement technique is shown in Fig. 3.

As shown in Fig. 3, after the excitation signal $(x(t))$ is generated with fixed frequency $\left(f_{i}\right)$, it is necessary to wait a certain number of settling time cycles before sampling the response signal $(y(t))$. The settling time cycles have significant effects on the impedance signatures, as discussed in Section V. Then, $N$ samples of the response signal are sampled, and the DFT with $N$ samples of the excitation and response signals are computed. For each frequency step $\left(d_{f}\right)$, the impedance of the transducer $\left(Z_{E}\left[f_{i}\right]\right)$ can be obtained by averaging several measurements to obtain impedance signatures with high accuracy and precision. To calculate the impedance according to (4), the input impedance $\left(Z_{\text {in }}\left[f_{i}\right]\right)$ of the DAQ device must be precalculated for each frequency step or measure following the procedure described above. Similarly, the excitation signal must be sampled and stored for each frequency step.

The time required for each iteration disregarding the DFT calculation is $\Delta t=N / f_{S}$, where $f_{S}$ is the sampling rate, in addition to the settling time. Depending on the analyzed frequency range defined by the initial $\left(f_{I}\right)$ and final frequency $\left(f_{F}\right)$, the frequency step, and the number of measurements for the average, many iterations may be required to obtain an impedance signature. Therefore, the steady-state measurement technique may require a long time to provide an impedance signature. The time required for the measurement is particularly critical in SHM systems in which the monitored structure operates under large temperature variations. It is well known that damage detection based on the EMI method is sensitive to temperature variations [1]. Temperature

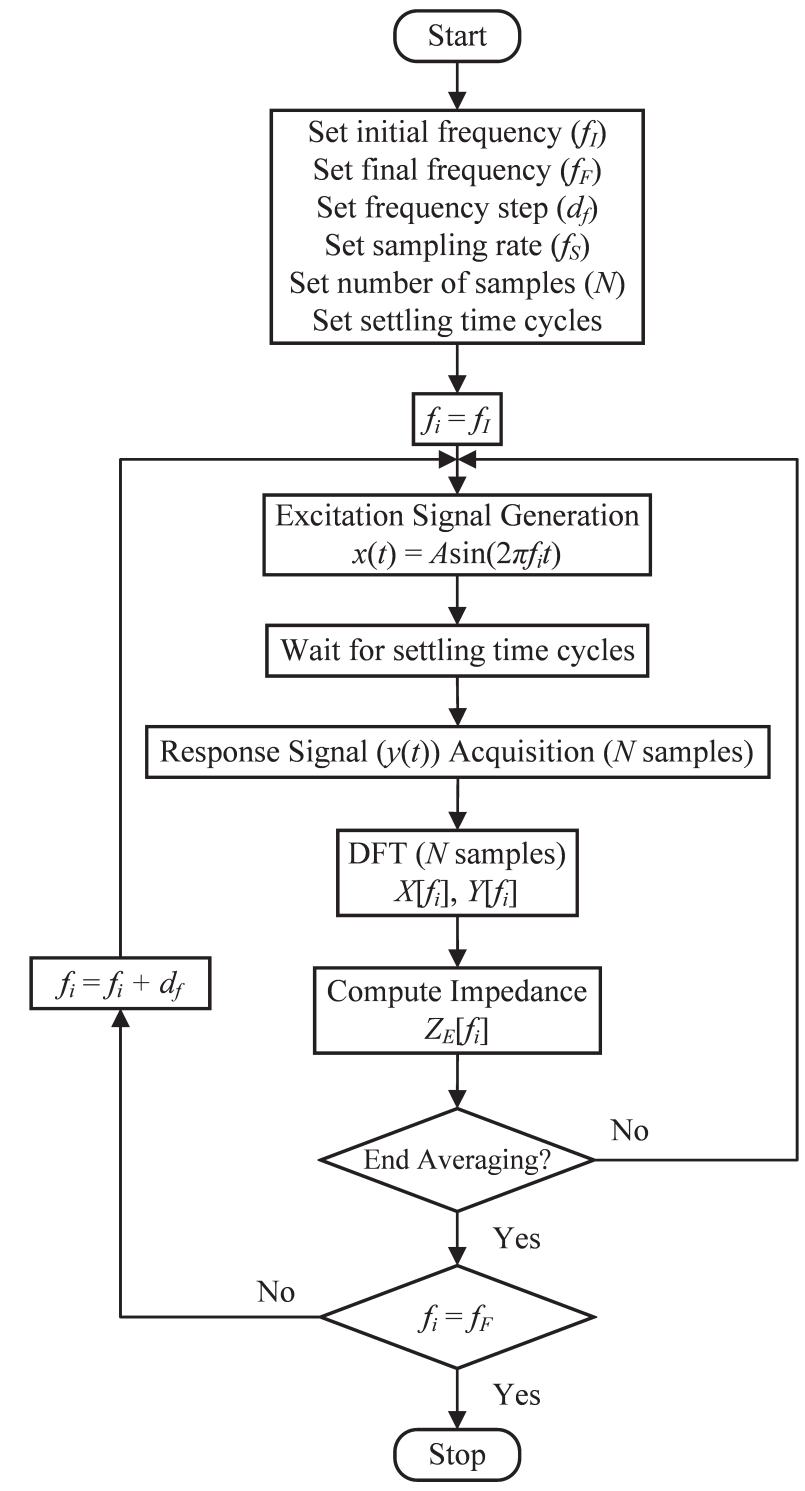

Fig. 3. Simplified flowchart of the steady-state measurement.

variations during the measurement process can unevenly alter impedance signatures, and techniques used to compensate for the effects of temperature are not always effective in this case. The time required for the measurement can be reduced using the transient-state measurement technique presented in Section III-B.

\section{B. Transient-State Measurement}

In the transient-state measurement, the excitation signal contains all the frequency components of the desired frequency range, allowing the impedance signatures to be obtained quickly. In this paper, this measurement technique was analyzed using the same system used for the analysis of the steady-state measurement technique, as shown in Fig. 2. However, instead of a sinusoidal signal, a dynamic signal was used as the excitation signal. The chirp is a dynamic signal that is straightforward to generate and widely used in many measurement systems [21], [27], [30], [31], [34]-[36]. A linear 


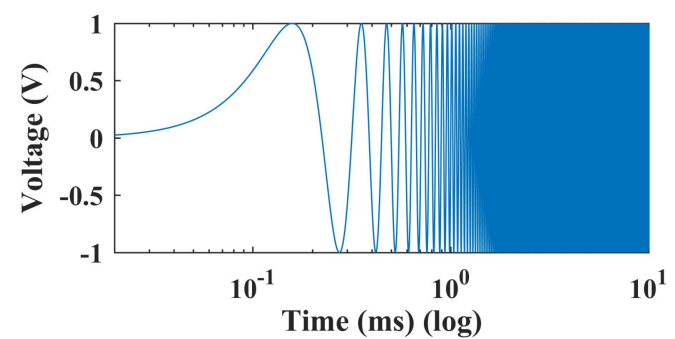

(a)

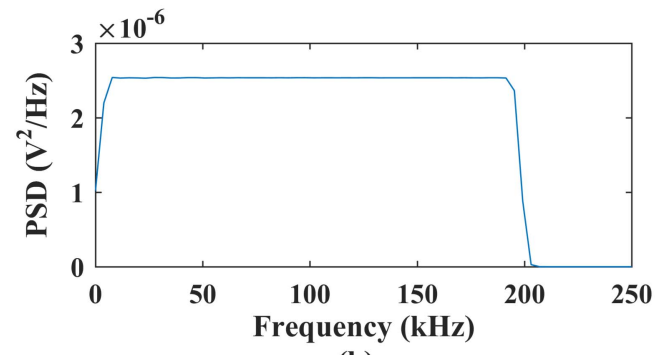

(b)

Fig. 4. (a) Waveform. (b) PSD of a chirp signal.

chirp was used as the excitation signal, where the frequency varies linearly with respect to time, given by

$$
x[n]=A \sin \left(\frac{2 \pi}{f_{S}} n\left(\frac{f_{F}-f_{I}}{2 N} n+f_{I}\right)\right)
$$

where $A$ is the amplitude, $f_{I}$ and $f_{F}$ are the initial and final frequency, respectively, $f_{S}$ is the sampling rate, $n$ is the sample, and $N$ is the number of samples.

As an example, Fig. 4(a) and (b) shows the waveform and the power spectral density (PSD) of a chirp signal with $A=1 \mathrm{~V}, N=20000, f_{I}=0, f_{F}=200 \mathrm{kHz}$, and $f_{S}=2 \mathrm{MS} / \mathrm{s}$.

According to Fig. 4, the frequency of the signal increases over time, and its spectrum contains all the frequencies of the desired range, which in this example is 0 to $200 \mathrm{kHz}$. Therefore, the transducer is excited over a wide frequency range in a short time, which is $\Delta t=N / f_{S}$. Consequently, the impedance signatures are obtained quickly, given by [21]

$$
Z_{T}[f]=R_{S} \frac{H[f]}{1-H[f]}
$$

where $H[f]$ is the frequency-response function (FRF), taking the excitation signal $(x[n])$ as the input and the response signal $(y[n])$ as the output. The FRF is computed by a spectral estimation given by

$$
H[f]=\frac{S_{x y}[f]}{S_{x x}[f]}
$$

where $S_{x y}[f]$ is the crossed power spectrum between the excitation signal $(x[n])$ and the response signal $(y[n])$ and $S_{x x}[f]$ is the autopower spectrum of the excitation signal, both obtained from the $X[f]$ and $Y[f]$ DFTs.

Similar to the description in Section III-A, the impedance $Z_{T}[f]$ calculated in (6) includes the input impedance $\left(Z_{\text {in }}[f]\right)$ effect of the DAQ device. The electrical impedance of the transducer $\left(Z_{E}[f]\right)$ can be obtained similarly, as indicated in (4). The simplified flowchart of this measurement technique is shown in Fig. 5.

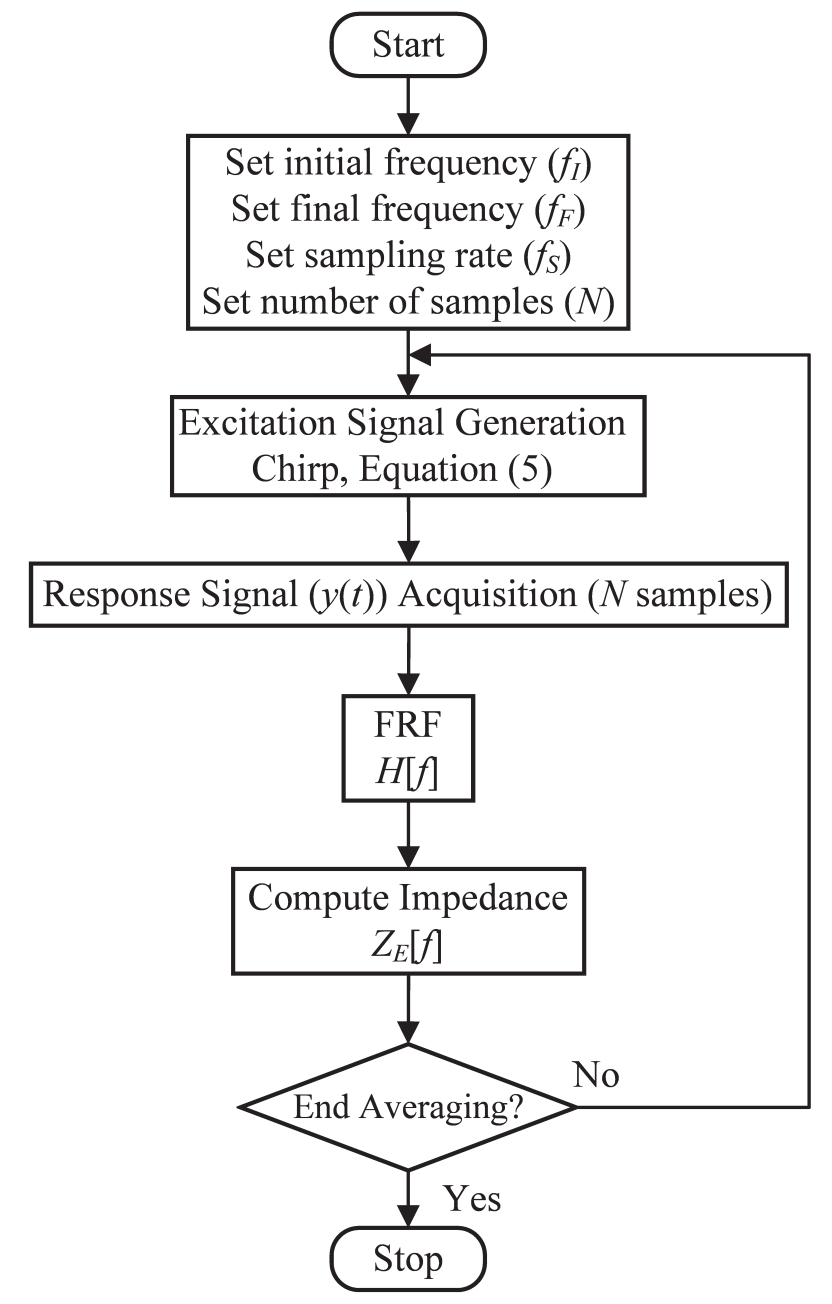

Fig. 5. Simplified flowchart of the transient-state measurement, adapted from [21].

As shown in the flowchart, the impedance signatures can be obtained by averaging several measurements. Unlike the technique described in Section III-A, the impedance signature over the entire desired frequency range in the transientstate measurement technique is obtained in a single iteration with the same duration as the chirp signal, disregarding the calculation of the FRF. Therefore, the measurement process is faster. A tradeoff exists between the sampling rate, the number of samples and the frequency step of the impedance signatures, which is given by $d_{f}=f_{S} / N$.

The two measurement techniques were analyzed and compared regarding the accuracy, precision, sensitivity to damage, and time required for the measurements according to the experimental configuration presented in Section IV.

\section{EXPERIMENTAL CONFIGURATION}

To analyze and compare the two measurement techniques, the system shown in Fig. 2 was implemented using an NI-USB-6361 DAQ device. The use of the same hardware permits an effective comparison between the two techniques, ensuring that the differences observed in the accuracy, precision, and time required for measurements are due to the technique and not to the hardware. A precision resistor of 


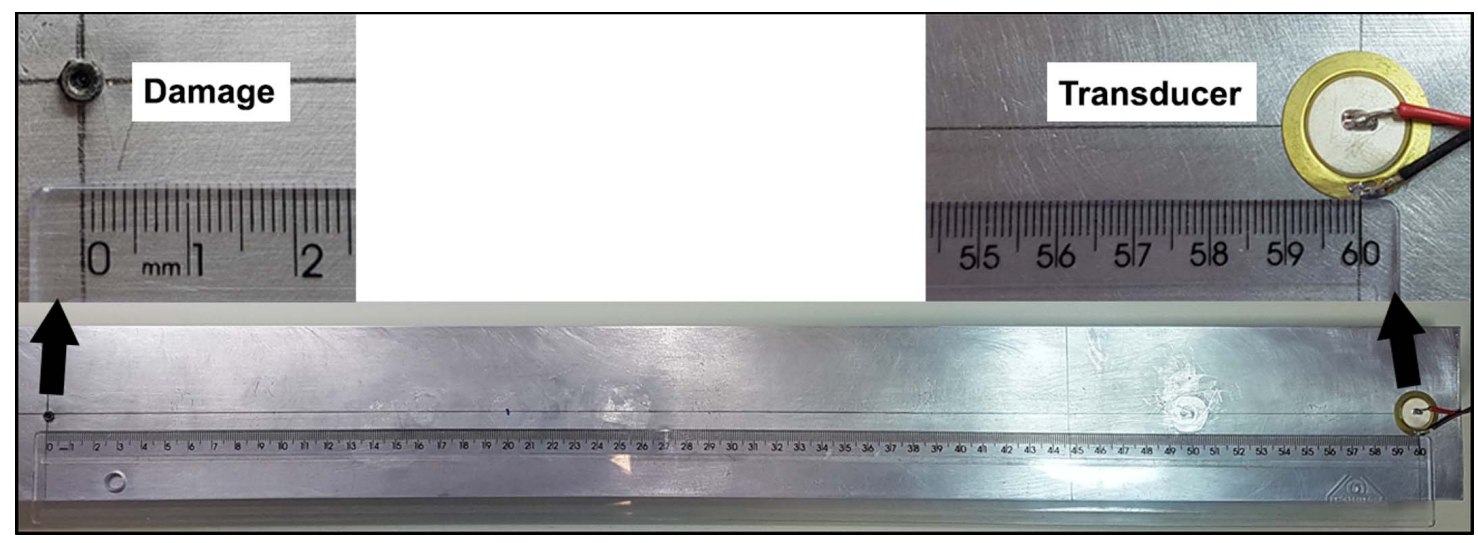

Fig. 6. Aluminum bar with piezoelectric transducer and induced damage.

$2.2 \mathrm{k} \Omega \pm 1 \%$ was used as the series resistor $\left(R_{S}\right)$ to connect the transducer or load under test. This value was chosen to ensure a low voltage and, consequently, a low electric field applied to the piezoelectric transducer. As is well known, the piezoelectric effect is approximately linear when the electric field is sufficiently low. The software for the two techniques was implemented in LabVIEW according to the flowcharts shown in Figs. 3 and 5.

For the steady-state measurement technique, sinusoidal signals with amplitude $A=1 \mathrm{~V}$ were used as excitation signals. The impedance signatures were obtained from $f_{I}=100 \mathrm{~Hz}$ to $f_{F}=200 \mathrm{kHz}$ with a step $d_{f}=100 \mathrm{~Hz}$ and sampling rate $f_{S}=2 \mathrm{MS} / \mathrm{s}$. These frequencies were selected according to the maximum sampling rate allowed by the DAQ device and the frequency response of the AI, which exhibits a flat response up to the frequency of $200 \mathrm{kHz}$. The DFTs were computed with 20000 samples of the excitation and response signals. As the measures are obtained step by step for fixed frequencies, the Hanning window was applied to the time signals before computing the DFTs, as shown in Fig. 2, to minimize harmonic frequency effects. By default, 1000 settling time cycles were used for each frequency step in most measurements, but other settings were also tested as discussed in Section V.

To apply the same configuration for the transient-state measurement technique, the chirp signal in (5) was set for the same frequency range, amplitude, sampling rate, and $N=$ 20000 samples, which resulted in impedance signatures with a frequency step of $100 \mathrm{~Hz}$. However, in contrast to the steadystate technique, the excitation and response signals contained all the frequencies of the range of interest, and the rectangular window was therefore used in the measurements.

In the first test, the two measurement techniques were compared using two basic loads: a resistor and a capacitor with nominal values of $100 \Omega$ and $10 \mathrm{nF}$, respectively. As reference values, measurements of an LCR meter, U1733C, from Agilent, configured for the $100 \mathrm{kHz}$ frequency, were used. The values obtained for the resistor and capacitor were $99.180 \Omega$ and $10.64 \mathrm{nF}$, respectively. In addition, the resistor has a small inductive component of $0.6 \mu \mathrm{H}$. Several impedance signatures were collected, and the mean and standard deviation were calculated to analyze and compare the precision of the two techniques and the accuracy based on the reference values.
Then, tests were performed on an aluminum bar with dimensions of $790 \mathrm{~mm} \times 75 \mathrm{~mm} \times 3 \mathrm{~mm}$ representing the monitored structure. A piezoelectric diaphragm, model 7BB20-6 manufactured by Murata Electronics North America, Inc. (Smyrna, GA, USA), with a circular brass plate of $20 \mathrm{~mm}$ in diameter and $0.20 \mathrm{~mm}$ in thickness and a piezoelectric ceramic (active element) of $14 \mathrm{~mm}$ in diameter and $0.22 \mathrm{~mm}$ in thickness, was fixed at $20 \mathrm{~mm}$ from one end of the bar using cyanoacrylate glue to obtain the impedance signatures. As demonstrated in previous experimental [30] and theoretical [31] studies, this piezoelectric diaphragm has properties similar to conventional PZT ceramics and is sensitive to damage, at least applied for small structures and excited with a lowvoltage signal, which are the experimental conditions used in this paper.

Damage was induced in the structure by mass addition by attaching a $2 \times 4 \mathrm{~mm}$ steel nut at a distance of $600 \mathrm{~mm}$ from the transducer using cyanoacrylate glue. This procedure alters the mechanical impedance of the structure in a similar way as actual damage, with the advantage of not permanently damaging the structure. This configuration is shown in Fig. 6.

The RMSD index was calculated using (2) in different frequency bands to analyze and compare the reproducibility of the results and sensitivity to the damage using the two measurement techniques. As is commonly known, the EMI method is sensitive to temperature variations [1]. Therefore, to avoid temperature effects when comparing the two measurement techniques, all measurements were taken at a controlled temperature of $25^{\circ} \mathrm{C}$ using an air conditioner. In addition, the aluminum bar was placed on rubber blocks on a workbench to minimize the effects of external vibrations. The experimental setup is shown in Fig. 7.

To ensure satisfactory accuracy, the input impedance of the DAQ device was considered as indicated in (4) in all measurements obtained by the two techniques. The results are presented and discussed in Section V.

\section{Results}

In this section, the results of the electrical impedances obtained for the basic loads and the transducer attached to the aluminum bar are presented and analyzed. The accuracy and 


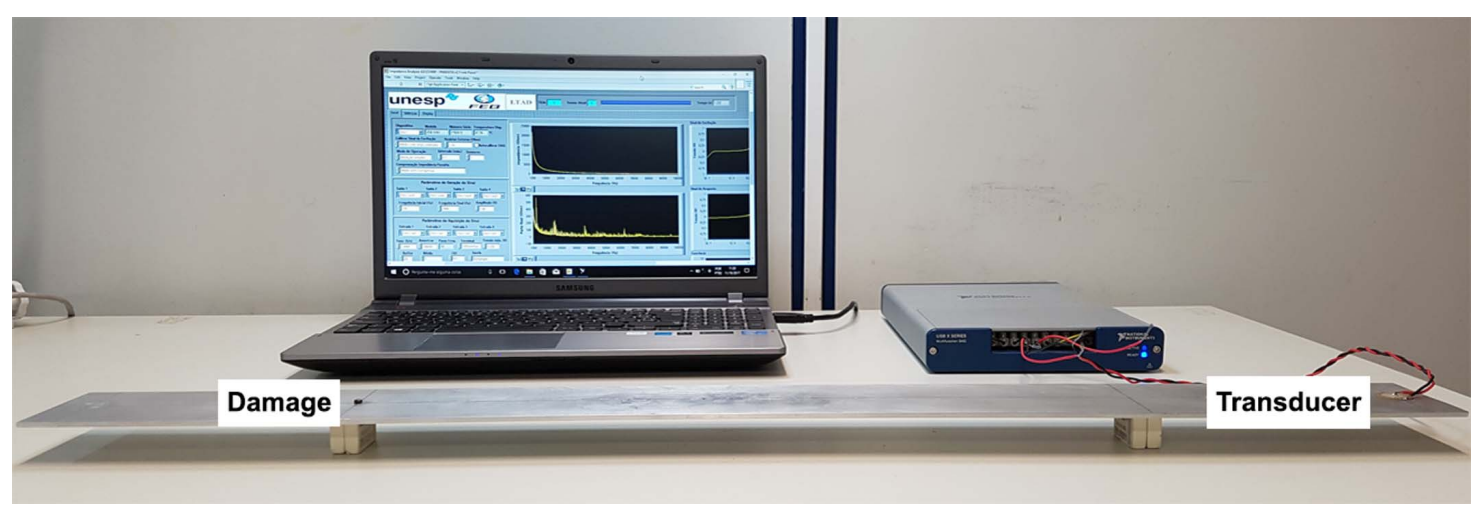

Fig. 7. Experimental setup.

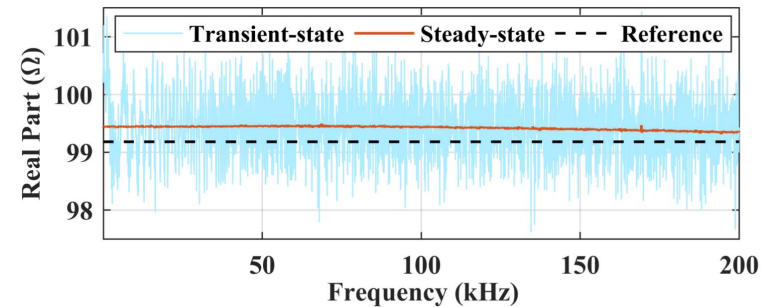

(a)

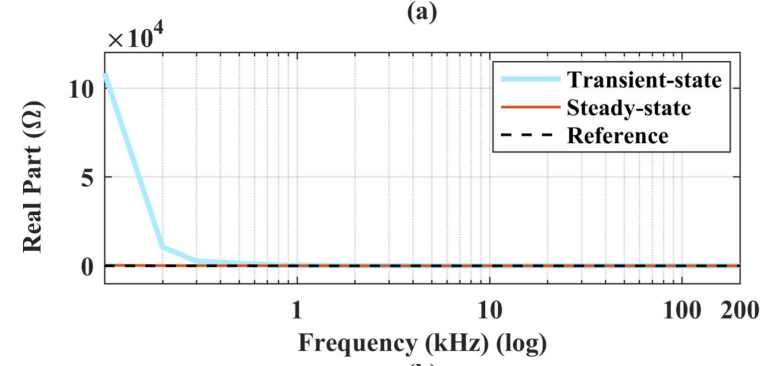

(b)

Fig. 8. Real part of the impedance obtained for the (a) resistor and (b) capacitor.

precision of the two measurement techniques were analyzed and compared, since the reproducibility of the results is important for impedance-based SHM systems to ensure the correct diagnosis of the monitored structure. The results obtained for the basic loads are presented in Section V-A.

\section{A. Basic Loads}

The electrical impedance signatures of the basic loads were collected using the two measurement techniques. The accuracy of the two techniques was analyzed based on the reference values obtained with a commercial instrument, as described in Section IV. The real and imaginary parts of the impedance signatures obtained for the resistor and capacitor are shown in Figs. 8(a) and (b) and 9(a) and (b), respectively. The magnitude of the impedance is not shown because it is simply a combination of the real and imaginary parts.

In addition to the impedance signatures obtained by the two measurement techniques, reference signatures based on the values obtained with the commercial instrument are also shown in Figs. 8 and 9. Note that the reference values were

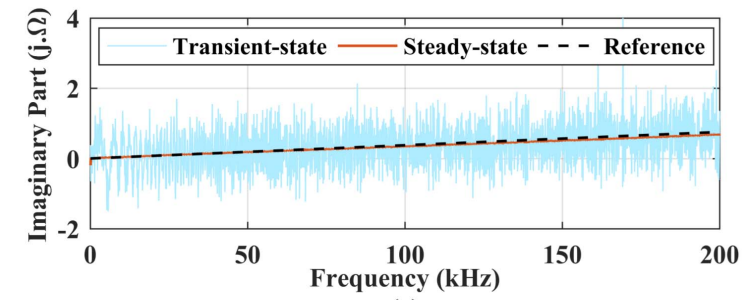

(a)

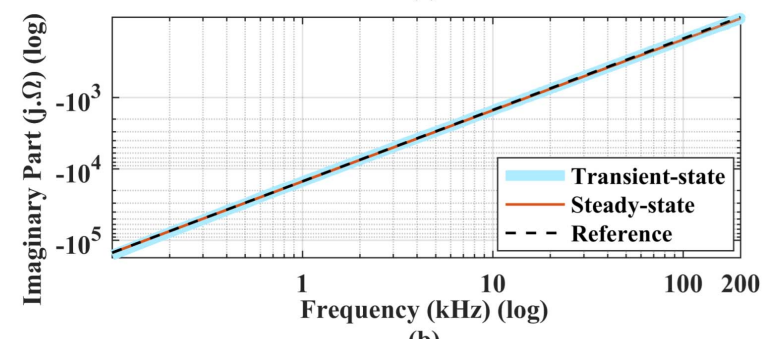

(b)

Fig. 9. Imaginary part of the impedance obtained for the (a) resistor and (b) capacitor.

obtained at the test frequency of $100 \mathrm{kHz}$, which is the highest test frequency allowed by the LCR meter (Agilent U1733C) used in this paper; for the other frequencies, the values were theoretically estimated. The signatures were obtained without averaging to ensure an effective comparison between the two techniques.

According to the results obtained for the resistive and capacitive loads, the two measurement techniques provide measurements close to the reference values. The imaginary part of the resistive load exhibits a slight increase with the frequency due to its small inductive component of $0.6 \mu \mathrm{H}$, as mentioned in Section IV. However, the measurements obtained using the steady-state technique are closer to the reference values and vary significantly less between the frequency steps than the measurements obtained using the transient-state technique. The largest discrepancy was observed for the real part of the impedance of the capacitor obtained using the transient-state technique, for which low values were expected but high values were obtained for frequencies below $300 \mathrm{~Hz}$. The lower the capacitance is, the more significant the discrepancy. However, this is not relevant for SHM applications because damage is 


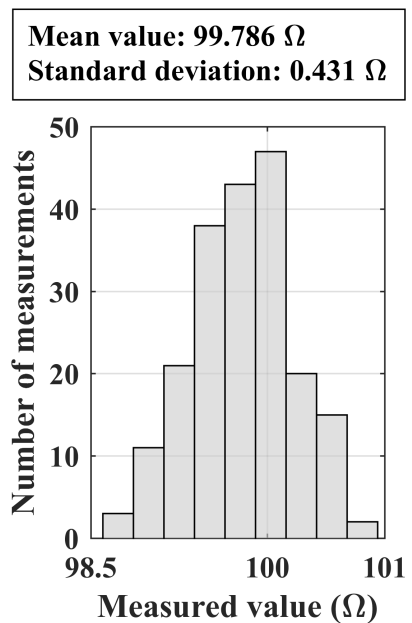

(a)
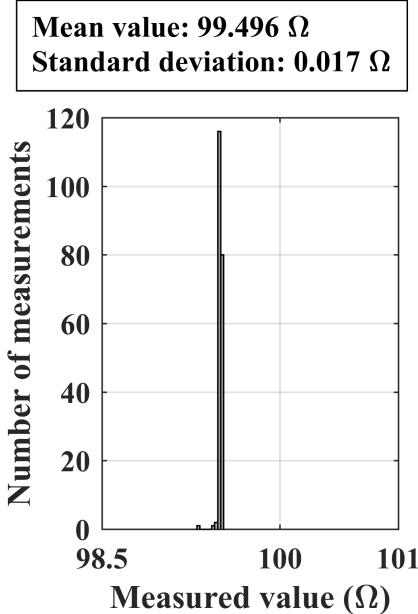

(b)
Fig. 10. Histograms of the measures obtained for the resistive load without averaging using the (a) transient-state and (b) steady-state techniques.

usually detected at high frequencies where the results are close to the reference values.

Another important feature that must be considered is the time required to obtain each impedance signature. The transient-state technique provides signatures relatively quickly, requiring only approximately $0.4 \mathrm{~s}$ for the configuration used in this paper. In contrast, the steady-state technique requires a much longer time of approximately $3.3 \mathrm{~min}$ to provide impedance signatures in the same range and frequency step, although the quality of the corresponding measurements is better, as shown in Figs. 8 and 9.

For a quantitative comparison between the two techniques, a more detailed analysis was performed considering the resistive load. Therefore, 200 impedance signatures were acquired, and the values of the resistance at a frequency of $100 \mathrm{kHz}$ were considered for the analysis, allowing the histogram of the measurements, the mean value and the standard deviation to all be obtained. Initially, the analysis was performed without averaging the impedance signatures. Fig. 10 shows the histograms of the measurements obtained for the two techniques.

As shown in Fig. 10, the measures obtained by the transientstate technique show significant dispersion in relation to the mean value of $99.786 \Omega$, with an error of $0.61 \%$ relative to the reference value $(99.180 \Omega)$ and a standard deviation of $0.431 \Omega$. In contrast, the measures provided by the steadystate technique are significantly more concentrated around the mean value of $99.496 \Omega$, with a $0.32 \%$ error relative to the reference value and standard deviation of only $0.017 \Omega$. Therefore, although the steady-state technique is significantly more precise (with a lower standard deviation), the two techniques have similar accuracy.

The accuracy and precision can be improved by taking an average of several measurements. As an example, Fig. 11 shows the histogram of 200 measures, where each measure was obtained as an average of 50 measurements.

According to the results, averaging enables a significant improvement in the precision. Fig. 11 shows that the measures

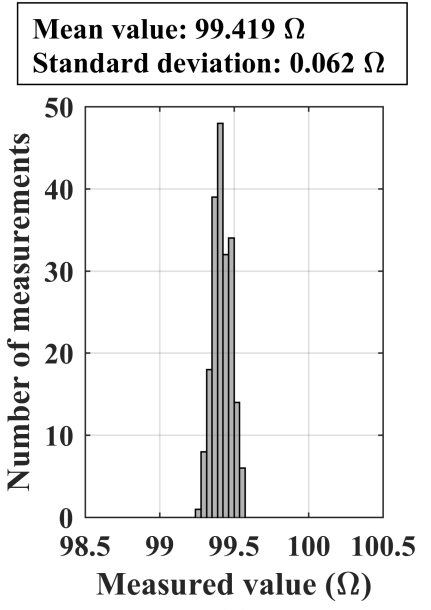

(a)

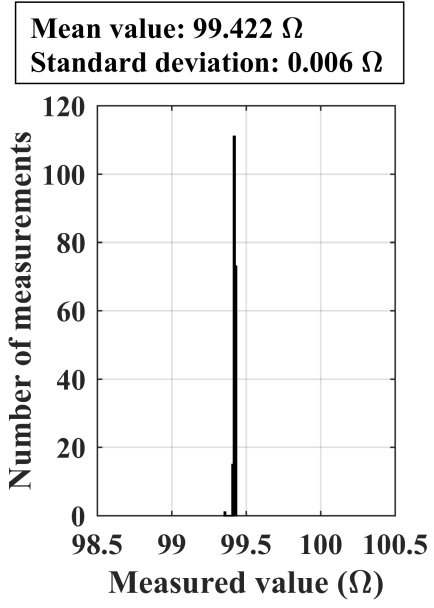

(b)
Fig. 11. Histograms of the measures obtained for the resistive load by averaging 50 measurements obtained using the (a) transient-state and (b) steady-state techniques.

obtained for the transient-state technique are more concentrated in relation to the mean value than the results obtained without averaging. By averaging the measurements, the standard deviation decreased from 0.431 to $0.062 \Omega$. For the steady-state technique, the standard deviation decreased from 0.017 to $0.006 \Omega$. The mean values also showed a small change due to the average of the measurements, becoming closer between the two techniques.

The effects of the averaging on the mean value and the standard deviation can be better analyzed using Fig. 12, which shows the variation of these parameters with respect to the number of measurements taken for the average.

According to Fig. 12, the standard deviation decreases (i.e., the precision increases) as the number of measurements used in averaging increases, converging to a minimum value, which occurred after 50 measurements for the system used in this paper. The mean value also exhibits a slight variation with the averaging, as noted earlier, indicating a similar accuracy for the two measurement techniques. The results obtained for the resistive load using the two measurement techniques are summarized in Table I.

As noted in Table I, although the two techniques provide measurements with similar accuracy, the precision between them is significantly different. The steady-state technique provides better precision, but requires much more time to provide an impedance signature. For example, for an average of 50 measurements, the steady-state technique requires approximately $2.8 \mathrm{~h}$ to provide an impedance signature with a precision of $\pm 0.006 \Omega$. In contrast, the transient-state technique requires only approximately $0.9 \mathrm{~s}$, although the precision is worse $( \pm 0.062 \Omega)$.

Therefore, a tradeoff exists between the precision and time required for the measurement. This tradeoff is particularly critical in SHM applications. A fast measurement technique is desirable if the structural monitoring requires that the impedance signatures be obtained over a wide range and small frequency step. However, a precise system allows the 
TABLE I

Comparison of the Results Obtained For the Two Measurement Techniques

\begin{tabular}{ccccc}
\hline \hline & \multicolumn{2}{c}{ Transient-state technique } & \multicolumn{2}{c}{ Steady-state technique } \\
\hline & No average & $\begin{array}{c}\text { Average with 50 } \\
\text { measurements }\end{array}$ & No average & $\begin{array}{c}\text { Average with 50 } \\
\text { measurements }\end{array}$ \\
\hline Time required & 0.4 seconds & 0.9 seconds & 3.3 minutes & 2.8 hours \\
Mean value & $99.786 \Omega$ & $99.419 \Omega$ & $99.496 \Omega$ & $99.422 \Omega$ \\
Accuracy & $99.389 \%$ & $99.759 \%$ & $99.681 \%$ & $99.756 \%$ \\
Precision & $\pm 0.431 \Omega$ & $\pm 0.062 \Omega$ & $\pm 0.017 \Omega$ & $\pm 0.006 \Omega$ \\
\hline \hline
\end{tabular}

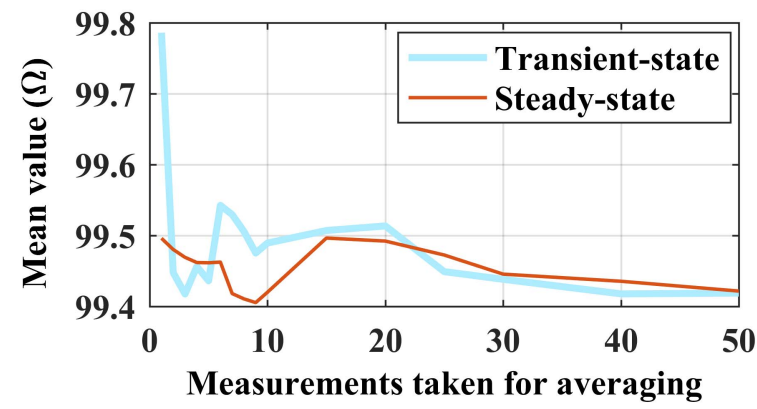

(a)

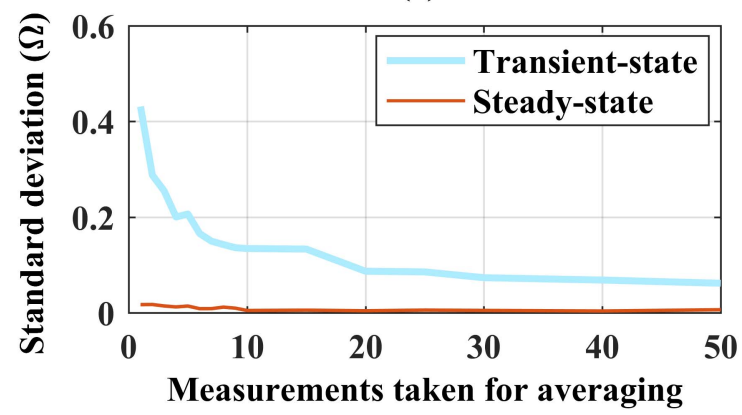

(b)

Fig. 12. Behavior of the (a) mean value and (b) standard deviation with respect to the number of measurements taken for the average.

reliable detection of incipient damage. The results obtained for the piezoelectric transducer fixed to the aluminum bar are presented in Section V-B.

\section{B. Piezoelectric Transducer}

The impedance signatures of the piezoelectric transducer were obtained using the same configuration used for the basic loads. A comparison of the real part and the imaginary part of the impedance signatures obtained using the two measurement techniques is shown in Fig. 13.

The impedances in Fig. 13 were obtained without averaging for an effective comparison between the two techniques. A narrowband of $90-110 \mathrm{kHz}$ is displayed for proper comparison between the signatures. For both the real part and the imaginary part, the signatures obtained using the two techniques have similar characteristics in amplitude and shape. The resonance peaks related to the natural frequencies of the structure, which are fundamental for the detection of damage, were correctly detected in the electrical impedance signatures obtained using the two techniques.

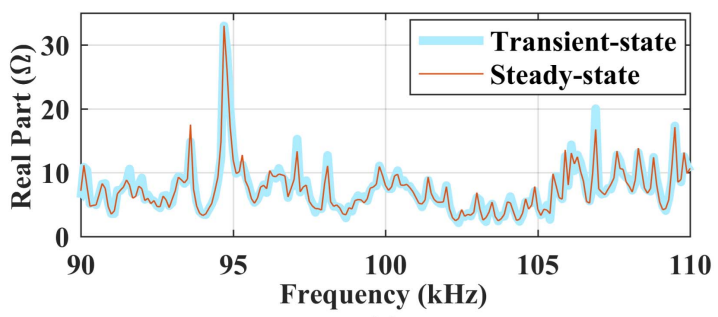

(a)

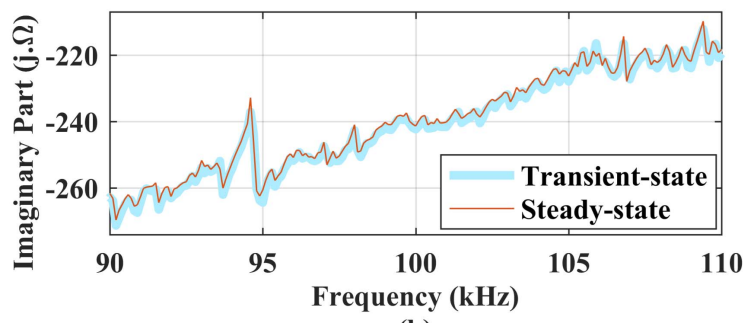

(b)

Fig. 13. (a) Real part and (b) imaginary part of the impedance signature obtained for the piezoelectric transducer using the two measurement techniques.

1) Reproducibility of the Results: As noted earlier, reproducibility of the results is important in impedance-based SHM applications because the damage detection is based on the comparison between two impedance signatures using damage indices. Variations in the impedance caused by the measurement system may make it challenging to diagnose the structure correctly. To evaluate the two measurement techniques regarding the reproducibility of the results, 11 impedance signatures were obtained without averaging. The first signature was used as the baseline in relation to the other signatures to calculate the RMSD index using (2). The RMSD index was calculated in subbands of $10 \mathrm{kHz}$ to evaluate the reproducibility of the results with respect to frequency. The RMSD indices are shown in Fig. 14.

As the monitored structure (aluminum bar) has not undergone any change due to damage and the measurements were taken in a temperature-controlled environment, low values are expected in the indices. According to Fig. 14, the indices obtained using the steady-state technique generally have lower values and lower variation between the frequency bands, indicating better reproducibility of the results relative to the transient-state technique. This result was expected since the results obtained for the basic loads presented in Section V-A indicated a better precision for the steady-state 


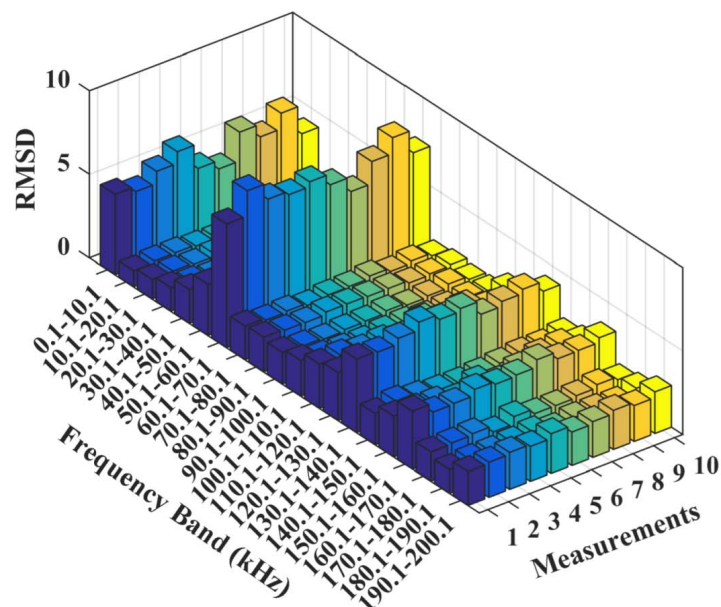

(a)

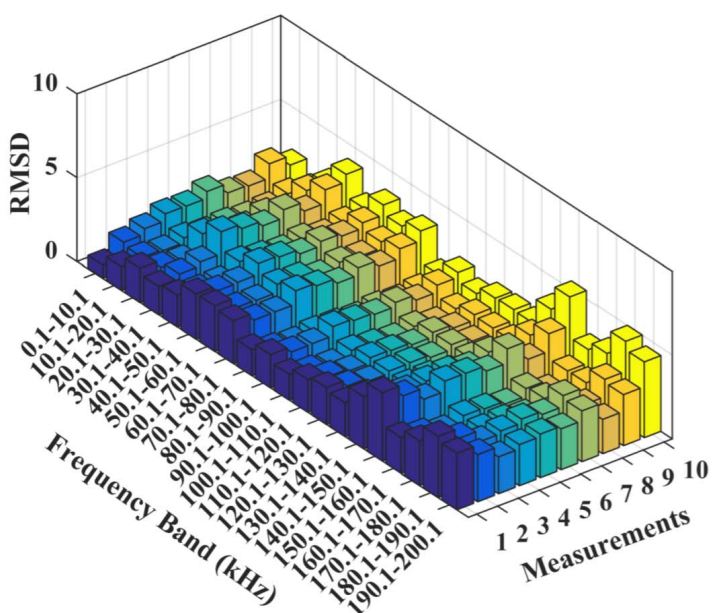

(b)

Fig. 14. RMSD indices obtained using the (a) transient-state and (b) steady-state techniques without averaging.

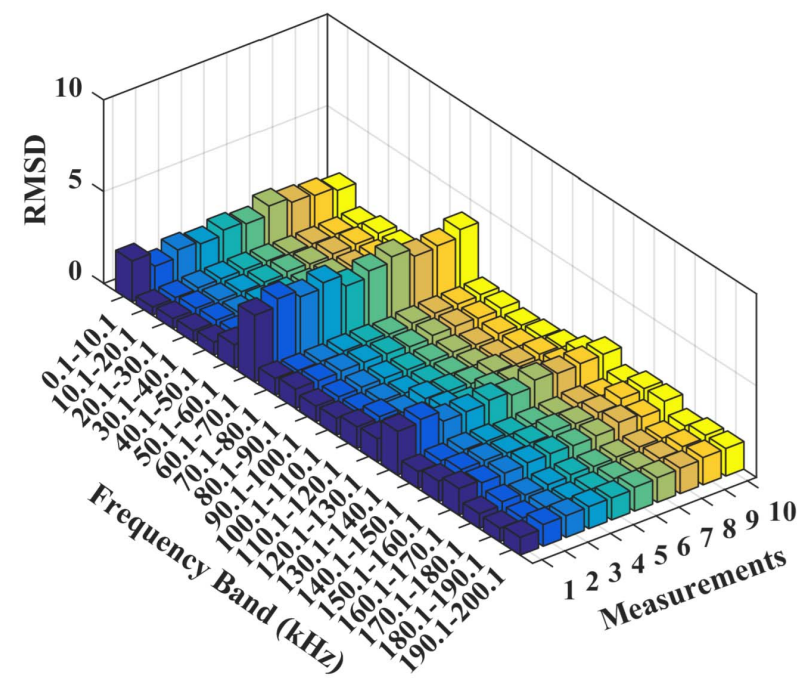

Fig. 15. RMSD indices obtained using the transient-state technique and taking an average of ten measurements.

technique. However, the reproducibility of the transient-state technique can be improved by averaging. As an example, Fig. 15 shows the indices obtained using the transient-state technique and impedance signatures obtained by taking an average of 10 measurements.

As shown in Fig. 15, performing the average clearly improves the reproducibility of the transient-state technique. In fact, the results are better than those obtained for the steadystate technique in certain frequency bands, with the advantage that the time required for measurements is much lower. The time required to obtain the results shown in Fig. 15 was $2.4 \mathrm{~s}$. The same results would require a time of $6.1 \mathrm{~h}$ using the steady-state technique.

2) Sensitivity to Structural Damage: In addition to the reproducibility of the results, it is important to analyze the sensitivity of each measurement technique to structural damage. Therefore, damage was induced in the structure following the

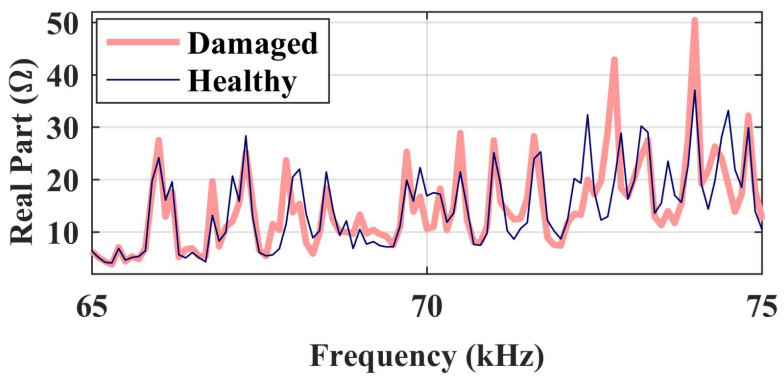

(a)

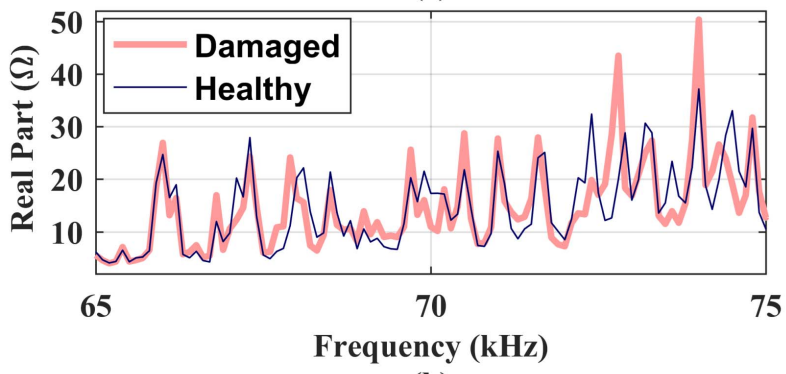

(b)

Fig. 16. Impedance signatures obtained for the structure under healthy and damaged conditions using the (a) transient-state and (b) steady-state measurement techniques.

procedure shown in Section IV, and the impedance signatures were obtained for the structure under the healthy and damaged conditions to calculate the RMSD index. The sensitivity to damage can be qualitatively evaluated by observing the variation that the damage causes in the impedance signatures. The impedance signatures obtained using the two measurement techniques for the structure under healthy and damaged conditions are shown in Fig. 16.

A narrow range of the impedance signatures more sensitive to the type of induced damage is shown in Fig. 16 for a better comparison. Variations due to the damage are observed in the impedance signatures obtained using the two measurement techniques, primarily in the range of $65-75 \mathrm{kHz}$. Therefore, 


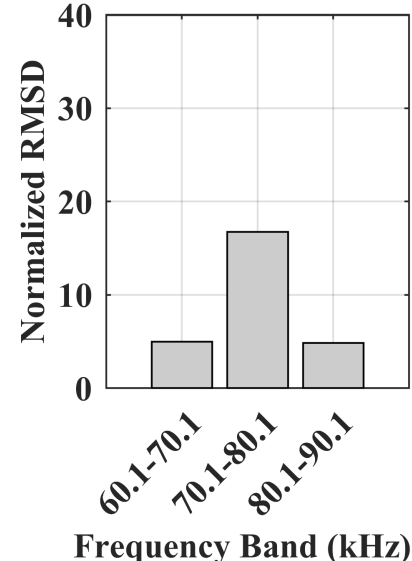

(a)

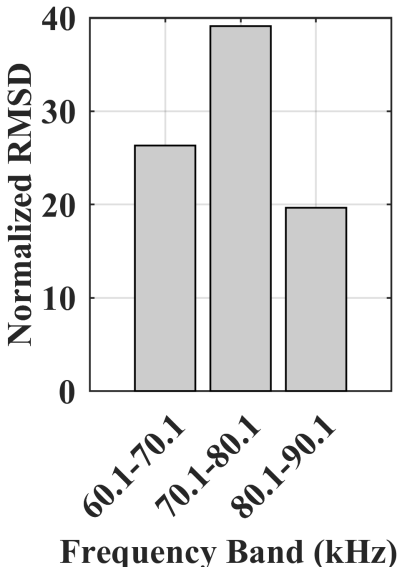

(b)
Fig. 17. RMSD indices obtained using the (a) transient-state technique and (b) steady-state technique.

this result indicates that both techniques are sensitive to structural damage. A quantitative analysis can be obtained by calculating the RMSD index from the signatures shown in Fig. 16. The index was calculated on subbands of $10 \mathrm{kHz}$, and the results are shown in Fig. 17.

The RMSD indices in Fig. 17 are normalized relative to the index obtained for the healthy structure, which was calculated by taking two impedance signatures without inducing damage. This approach allows one to analyze the real variation in the index due to structural damage. According to the results, the steady-state measurement technique is more sensitive to damage than the transient-state technique, providing RMSD indices with higher values. However, the results of the transient-state technique can be improved by performing averaging, as indicated by the previous results.

3) Settling Time Cycles: For SHM applications, an important tradeoff exists between the numbers of settling time cycles and the samples taken to compute the DFT, as shown in the flowchart of Fig. 3. The total time over which the monitored structure is excited, which is the sum of the times required by the settling time cycles and acquisition of the samples to compute the DFT, must be sufficient to detect the resonance peaks at the impedance signatures related to the natural frequencies of the structure.

This issue is particularly critical in embedded systems [22]-[26] in which the number of samples acquired to compute DFT is limited, requiring a greater number of settling time cycles. To exemplify this issue, a new test was performed by changing the number of samples in the steadystate measurement technique to $N=1024$, which is a common quantity in commercial devices. In addition, the frequency step was reduced to $10 \mathrm{~Hz}$ for better observation of the resonance peaks. Fig. 18 shows a comparison between the impedance signatures obtained using the two measurement techniques for the number of settling time cycles ranging from 0 to 1000 .

According to Fig. 18, the settling time cycles have a significant influence on the impedance signatures. Without settling time cycles, the resonance peaks are not detected in

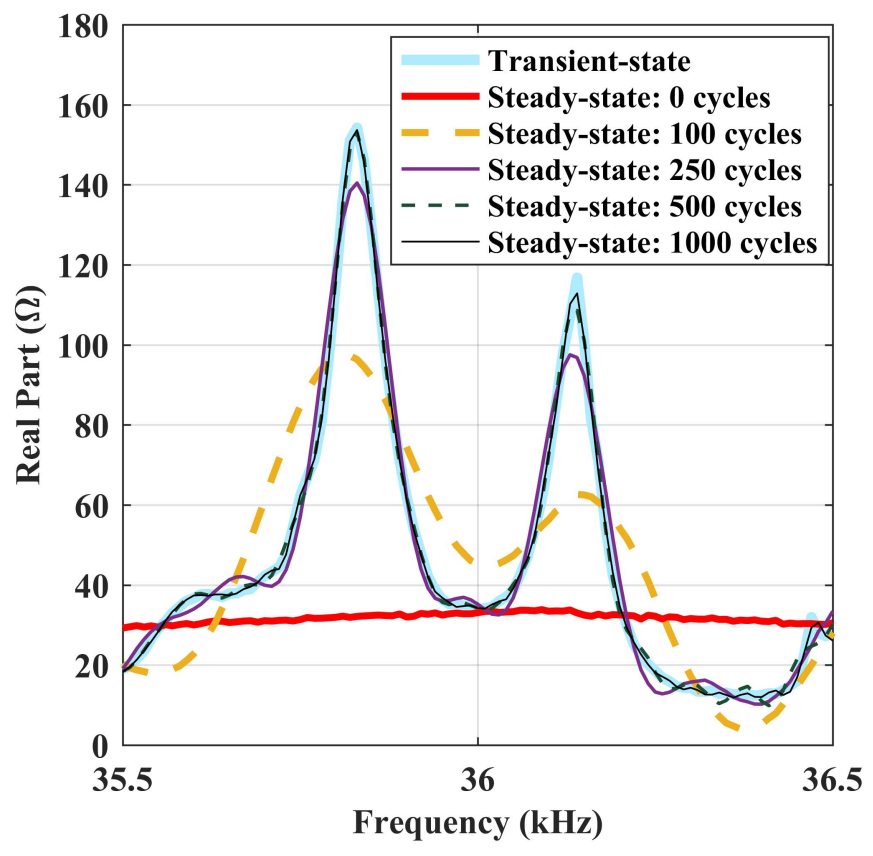

Fig. 18. Effects of the settling time cycles of the steady-state measurement technique on the impedance signatures.

the impedance signatures, which makes it impossible to detect structural damage. The resonance peaks in the impedance signatures obtained by the steady-state technique approximate those obtained using the transient-state technique with 1000 settling time cycles. Fig. 18 shows the impedance signatures over a narrow frequency range at approximately $36 \mathrm{kHz}$, but similar results are obtained over the entire frequency range.

The transient-state technique has an advantage in this context. Since the excitation signal has a wide frequency band, as shown in Fig. 4, all natural frequencies of the monitored structure are excited over a short time interval, rapidly providing the impedance signatures.

\section{CONCLUSION}

This paper presents a comparative study of two measurement techniques commonly used in impedance-based SHM applications, which are the steady-state and transientstate techniques. Tests were performed on basic loads and a transducer attached to an aluminum bar representing the monitored structure. The results reveal conclusively important differences between the two techniques regarding the precision, sensitivity to structural damage, and the time required for the measurements.

Although the two techniques exhibit similar accuracy, the steady-state technique is more precise and sensitive to damage. In contrast, the transient-state technique allows fast measurements, and its precision can be improved by averaging the measurements, as indicated by the experimental results. The steady-state technique requires settling time cycles for the correct detection of the resonance peaks in the impedance signatures related to the natural frequencies of the structure, which requires more time to obtain measurements and is a disadvantage relative to the transient-state technique. 
Therefore, an important tradeoff exists between the precision and the time required for the measurements that must be taken into account in impedance-based SHM and other applications. The results presented in this paper provide a reference for choosing the appropriate measurement technique or development approach for new techniques in SHM and other applications, depending on the severity of the damage to be detected, range and frequency step over which the impedance signatures are measured and the hardware used for the measurements.

In this paper, the transient-state measurement technique was analyzed based on the chirp signal, which is a wellknown signal that is widely used for transducer excitation. However, other types of signals, such as impulsive signals, can be analyzed in the future research and compared with conventional signals. In addition to the accuracy, precision, sensitivity to damage, and time required for measurements, other characteristics, such as power consumption and the signal-to-noise ratio, can be analyzed.

\section{ACKNOWLEDGMENT}

The authors would like to thank the anonymous reviewers for their valuable recommendations that helped improved this article.

\section{REFERENCES}

[1] C. R. Farrar and K. Worden, Structural Health Monitoring: A Machine Learning Perspective. Hoboken, NJ, USA: Wiley, 2013.

[2] H.-N. Li, L. Ren, Z.-G. Jia, T.-H. Yi, and D.-S. Li, "State-of-the-art in structural health monitoring of large and complex civil infrastructures," J. Civil Struct. Health Monitor, vol. 6, no. 1, pp. 3-16, Feb. 2016.

[3] J. Song, S. Kim, Z. Liu, N. N. Quang, and F. Bien, "A real time nondestructive crack detection system for the automotive stamping process," IEEE Trans. Instrum. Meas., vol. 65, no. 11, pp. 2434-2441, Nov. 2016.

[4] M. D. Prieto et al., "Self-powered wireless sensor applied to gear diagnosis based on acoustic emission," IEEE Trans. Instrum. Meas., vol. 65, no. 1, pp. 15-24, Jan. 2016.

[5] E. Ramasso, V. Placet, and M. L. Boubakar, "Unsupervised consensus clustering of acoustic emission time-series for robust damage sequence estimation in composites," IEEE Trans. Instrum. Meas., vol. 64, no. 12 , pp. 3297-3307, Dec. 2015.

[6] S. Shan, L. Cheng, and P. Li, "Adhesive nonlinearity in Lamb-wavebased structural health monitoring systems," Smart Mater. Struct., vol. 26, no. 2, p. 25019, 2017.

[7] X. Chen, X. Li, S. Wang, Z. Yang, B. Chen, and Z. He, "Composite damage detection based on redundant second-generation wavelet transform and fractal dimension tomography algorithm of Lamb wave," IEEE Trans. Instrum. Meas., vol. 62, no. 5, pp. 1354-1363, May 2013.

[8] T. J. Rocha, H. G. Ramos, A. L. Ribeiro, and D. J. Pasadas, "Evaluation of subsurface defects using diffusion of motion-induced eddy currents," IEEE Trans. Instrum. Meas., vol. 65, no. 5, pp. 1182-1187, May 2016.

[9] X. Li, B. Gao, W. L. Woo, G. Y. Tian, X. Qiu, and L. Gu, "Quantitative surface crack evaluation based on eddy current pulsed thermography," IEEE Sensors J., vol. 17, no. 2, pp. 412-421, Jan. 2017.

[10] J.-M. Muñoz-Ferreras, Z. Peng, Y. Tang, R. Gómez-García, D. Liang, and C. Li, "Short-range Doppler-radar signatures from industrial wind turbines: Theory, simulations, and measurements," IEEE Trans. Instrum. Meas., vol. 65, no. 9, pp. 2108-2119, Sep. 2016.

[11] H. Z. Hosseinabadi, B. Nazari, R. Amirfattahi, H. R. Mirdamadi, and A. R. Sadri, "Wavelet network approach for structural damage identification using guided ultrasonic waves," IEEE Trans. Instrum. Meas., vol. 63, no. 7, pp. 1680-1692, Jul. 2014.

[12] H. Cao et al., "Development and characterization of a novel interdigitated capacitive strain sensor for structural health monitoring," IEEE Sensors J., vol. 15, no. 11, pp. 6542-6548, Nov. 2015.

[13] S. A. A. Jabir and N. K. Gupta, "Thick-film ceramic strain sensors for structural health monitoring," IEEE Trans. Instrum. Meas., vol. 60, no. 11, pp. 3669-3676, Nov. 2011.
[14] K. Perveen, G. E. Bridges, S. Bhadra, and D. J. Thomson, "Corrosion potential sensor for remote monitoring of civil structure based on printed circuit board sensor," IEEE Trans. Instrum. Meas., vol. 63, no. 10, pp. 2422-2431, Oct. 2014.

[15] M. I. Albakri and P. A. Tarazaga, "Electromechanical impedance-based damage characterization using spectral element method," J. Intell. Mater. Syst. Struct., vol. 28, no. 1, pp. 63-77, 2017.

[16] P. Liu, W. Wang, Y. Chen, X. Feng, and L. Miao, "Concrete damage diagnosis using electromechanical impedance technique," Construction Building Mater, vol. 136, pp. 450-455, Apr. 2017.

[17] V. G. Annamdas and M. A. Radhika, "Electromechanical impedance of piezoelectric transducers for monitoring metallic and non-metallic structures: A review of wired, wireless and energy-harvesting methods," J. Intell. Mater. Syst. Struct., vol. 24, no. 9, pp. 1021-1042, 2013.

[18] W. Roth and V. Giurgiutiu, "Structural health monitoring of an adhesive disbond through electromechanical impedance spectroscopy," Int. J. Adhesion Adhesives, vol. 73, pp. 109-117, Mar. 2017.

[19] C. Rugina, D. Enciu, and M. Tudose, "Numerical and experimental study of circular disc electromechanical impedance spectroscopy signature changes due to structural damage and sensor degradation," Struct. Health Monitor., vol. 14, no. 6, pp. 661-663, 2015.

[20] T. Piasecki, K. Chabowski, and K. Nitsch, "Design, calibration and tests of versatile low frequency impedance analyser based on ARM microcontroller," Measurement, vol. 91, pp. 155-161, Sep. 2016.

[21] F. G. Baptista and J. V. Filho, "A new impedance measurement system for PZT-based structural health monitoring," IEEE Trans. Instrum. Meas., vol. 58, no. 10, pp. 3602-3608, Oct. 2009.

[22] (Apr. 2017). AD5933 Data Sheet. Analog Devices. Norwood, MA, USA. [Online]. Available: http://www.analog.com/media/en/technicaldocumentation/data-sheets/AD5933.pdf

[23] W. S. Na and K.-T. Park, "A cost-effective impedance-based structural health monitoring technique for steel structures by monitoring multiple areas," J. Intell. Mater. Syst. Struct., vol. 28, no. 2, pp. 154-162, Jan. 2017.

[24] A. Martowicz, A. Sendecki, M. Salamon, M. Rosiek, and T. Uhl, "Application of electromechanical impedance-based SHM for damage detection in bolted pipeline connection," Nondestruct. Test. Eval., vol. 31, no. 1, pp. 17-44, Jan. 2016.

[25] R. Harder, A. Diedrich, J. S. Whitfield, M. S. Buchowski, J. B. Pietsch, and F. J. Baudenbacher, "Smart multi-frequency bioelectrical impedance spectrometer for BIA and BIVA applications," IEEE Trans. Biomed. Circuits Syst., vol. 10, no. 4, pp. 912-919, Aug. 2016.

[26] X. Liu, C. Cheng, J. Wu, S. Eda, and Y. Guo, "A low cost and palm-size analyzer for rapid and sensitive protein detection by $\mathrm{AC}$ electrokinetics capacitive sensing," Biosensors Bioelectron., vol. 90 pp. 83-90, Apr. 2017.

[27] P. Slepski and K. Darowicki, "Optimization of impedance measurements using 'chirp' type perturbation signal," Measurement, vol. 42, no. 8, pp. 1220-1225, Oct. 2009.

[28] V. Giurgiutiu, "Piezoelectric wafer active sensors for structural health monitoring-state of the art and future directions," in Proc. Conf. Pressure Vessels Piping Division, Bellevue, WA, USA, 2010, pp. 1115-1121.

[29] S. Shahab and A. Erturk, "Coupling of experimentally validated electroelastic dynamics and mixing rules formulation for macro-fiber composite piezoelectric structures," J. Intell. Mater. Syst. Struct., vol. 28, no. 12, pp. 1575-1588, Jul. 2017.

[30] E. S. de Freitas and F. G. Baptista, "Experimental analysis of the feasibility of low-cost piezoelectric diaphragms in impedance-based SHM applications," Sens. Actuators A, Phys., vol. 238, pp. 220-228, Feb. 2016.

[31] E. S. de Freitas, F. G. Baptista, D. E. Budoya, and B. A. de Castro, "Equivalent circuit of piezoelectric diaphragms for impedance-based structural health monitoring applications," IEEE Sensors J., vol. 17, no. 17 , pp. 5537-5546, Sep. 2017.

[32] C. Liang, F. P. Sun, and C. A. Rogers, "Coupled electro-mechanical analysis of adaptive material systems-determination of the actuator power consumption and system energy transfer," J. Intell. Mater. Syst. Struct., vol. 8, no. 4, pp. 335-343, 1997.

[33] D. M. Peairs, D. J. Inman, and G. Park, "Circuit analysis of impedancebased health monitoring of beams using spectral elements," Structural Health Monitor, vol. 6, no. 1, pp. 81-94, 2007.

[34] S. G. Taylor, E. Y. Raby, K. M. Farinholt, G. Park, and M. D. Todd, "Active-sensing platform for structural health monitoring: Development and deployment," Struct. Health Monitor., vol. 15, no. 4, pp. 413-422, 2016. 
[35] E. L. M. Ribolla, P. Rizzo, and V. Gulizzi, "On the use of the electromechanical impedance technique for the assessment of dental implant stability: Modeling and experimentation," J. Intell. Mater. Syst. Struct., vol. 26, no. 16, pp. 2266-2280, 2015.

[36] V. Gulizzi, P. Rizzo, A. Milazzo, and E. L. M. Ribolla, "An integrated structural health monitoring system based on electromechanical impedance and guided ultrasonic waves," J. Civil Struct. Health Monitor, vol. 5, no. 3, pp. 337-352, Jul. 2015.

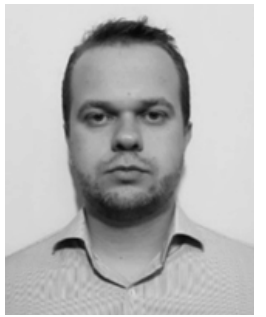

Danilo Ecidir Budoya (S'12-M'17) was born in Bauru, Brazil, in 1989. He received the B.S. degree in electrical engineering from São Paulo State University (UNESP), Bauru, in 2016, where he is currently pursuing the master's degree.

$\mathrm{He}$ is currently a Researcher with the Laboratory of Transducers and Data Acquisition, Department of Electrical Engineering, UNESP, focusing on structural health monitoring, signal processing, data acquisition, and electronic instrumentation.

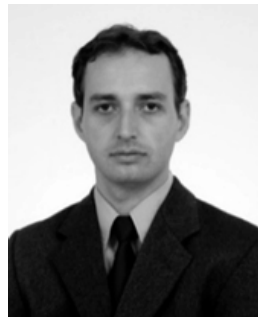

Fabricio Guimarães Baptista (S'08-M'11) received the B.S. and the Ph.D. degrees in electrical engineering from São Paulo State University (UNESP), Bauru, Brazil, in 2006 and 2010, respectively.

From 2010 to 2011, he was a Visiting Scholar at the Center for Intelligent Material Systems and Structures (CIMSS), Virginia Tech, Blacksburg,

VA, USA, focusing on structural health monitoring and piezoelectric transducers. $\mathrm{He}$ is currently an Assistant Professor with the Department of Electrical Engineering, UNESP, where he coordinates the Laboratory of Transducers and Data Acquisition, focusing on piezoelectricity, structural health monitoring, signal processing, data acquisition, and electronic instrumentation. 